\title{
THE SECOND MAIN THEOREM FOR HOLOMORPHIC CURVES INTERSECTING HYPERSURFACES WITH CASORATI DETERMINANT INTO COMPLEX PROJECTIVE SPACES
}

\author{
Tingbin Cao and Jun Nie
}

\author{
Nanchang University, Department of Mathematics \\ Nanchang, 330031, P. R. China; tbcao@ncu.edu.cn \\ Nanchang University, Department of Mathematics \\ Nanchang, 330031, P. R. China; jniemath@126.com
}

\begin{abstract}
Let $f: \mathbf{C} \rightarrow \mathbf{P}^{n}(\mathbf{C})$ be a holomorphic curves with hyperorder strictly less than 1 , and algebraically nondegenerate over the field $\mathcal{P}_{c}^{1}$ which consists of $c$-periodic meromorphic functions on $\mathbf{C}$. Let $\left\{Q_{j}\right\}_{j=1}^{q}$ be fixed or $c$-periodic slowly moving hypersurfaces with degree $d_{j}(j \in\{1, \ldots, q\})$ in (weakly) $N$-subgeneral position in $\mathbf{P}^{n}(\mathbf{C})$. In this paper, we prove a difference version of the second main theorem for $f$ intersecting $\left\{Q_{j}\right\}_{j=1}^{q}$ by using the Casorati determinant. A difference counterpart of the truncated second main theorem is also obtained. Our results extend the second main theorems for differences with fixed hyperplanes [9] or c-periodic slowly moving hyperplanes [10].
\end{abstract}

\section{Introduction and main results}

In 1925, Nevanlinna [11] established the value distribution theory for meromorphic functions in the complex plane $\mathbf{C}$, in which the second main theorem is the most important part. In 1933, Cartan [4] extended the Nevanlinna's second main theorem to the case for holomorphic curves sharing hyperplanes in general position into complex projective spaces. In 1983, Nochka [12] solved the Cartan's conjecture and extended the Cartan's second main theorem to the case for hyperplanes in subgeneral position. In 2004, Ru [17] extended the Cartan's second main theorem to the case of hypersurfaces. For the background of Nevanlinna theory, refer to see for examples $[16,14]$.

In the recent ten years, motivated by investigating the value distribution of complex difference polynomials and solutions of complex difference equations (refer to see [5]), the difference analogues of second main theorems were established. In 2006, Halburd and Korhonen [8] obtained the $c$-difference analogue of the second main theorem for meromorphic functions in the complex plane. Wong, Law and Wong [18] and Halburd, Korhonen and Tohge [9] have independently obtained the $c$-difference analogue of the second main theorem of holomorphic curves intersecting hyperplanes in general position into complex projective spaces. In 2016, Korhonen, Li and Tohge [10] continued to consider the second main theorem for the case of slowly moving

https://doi.org/10.5186/aasfm.2017.4259

2010 Mathematics Subject Classification: Primary 32H30; Secondary 30D35.

Key words: Holomorphic curve, hypersurfaces, second main theorem, complex projective space, Casorati determinant.

The paper was partially supported by NSFC (no. 11461042) and the NSF of Jiangxi Provence in China (no. 20161BAB201007). 
hyperplanes. Thus there rises a natural and interesting problem: What about the difference version of the second main theorem for fixed or slowly moving hypersurfaces into complex projective spaces?

Let $c \in \mathbf{C} \backslash\{0\}$, and let $f: \mathbf{C} \rightarrow \mathbf{P}^{n}(\mathbf{C})$ be a holomorphic map with the reduced representation $f=\left[f_{0}, \ldots, f_{n}\right]$. We use short notations

$$
f(z) \equiv f:=\bar{f}^{[0]}, f(z+c) \equiv \bar{f}=: \bar{f}^{[1]}, f(z+2 c) \equiv \overline{\bar{f}}=: \bar{f}^{[2]}, \cdots, f(z+k c) \equiv \bar{f}^{[k]} \text {. }
$$

Then similarly as the definition of the Wronskian determinant, the Casorati determinant of $f$ is defined by

$$
C(f)=C\left(f_{0}, \cdots, f_{n}\right)=\left|\begin{array}{cccc}
\frac{f_{0}}{f_{0}} & \frac{f_{1}}{f_{1}} & \cdots & \frac{f_{n}}{f_{n}} \\
\vdots & \vdots & \vdots & \vdots \\
{\frac{f_{0}}{[n]}}^{[n]} & \bar{f}_{1}^{[n]} & \cdots & {\overline{f_{n}}}^{[n]}
\end{array}\right| .
$$

Given a real positive integer $d$. Set $M=\left(\begin{array}{l}n+d \\ d\end{array}\right)-1, \mathcal{J}_{d}=\left\{\left(i_{0}, \cdots, i_{n}\right) \in \mathbf{N}_{0}^{n+1}: i_{0}+\right.$ $\left.\cdots+i_{n}=d\right\}$. For any $I_{j}=\left(i_{j 0}, \ldots, i_{j n}\right) \in \mathcal{J}_{d}, j \in\{0,1, \ldots, M\}$, set $f^{I_{j}}=f_{0}^{i_{j 0}} \cdots f_{n}^{i_{j n}}$. Then the Casorati determinant $\tilde{C}(f)=C\left(f^{I_{0}}, \ldots, f^{I_{M}}\right)$ is given as

$$
\tilde{C}(f)=C\left(f^{I_{0}}, \ldots, f^{I_{M}}\right)=\left|\begin{array}{cccc}
\frac{f^{I_{0}}}{f^{I_{0}}} & \frac{f^{I_{1}}}{f^{I_{1}}} & \ldots & \frac{f^{I_{M}}}{f^{I_{M}}} \\
\vdots & \vdots & \vdots & \vdots \\
\frac{f^{I_{0}}}{[M]} & \frac{f^{I_{1}}}{[M]} & \ldots & \frac{f^{I_{M}}}{[M]}
\end{array}\right| .
$$

Clearly, when $d=1$, we have $|\tilde{C}(f)|=|C(f)|$. Moreover, one can rearrange the order of $I_{0}, \ldots, I_{M}$ such that $\tilde{C}(f)=C(f)$ whenever $d=1$.

Denote by $T_{f}(r)$ the Nevanlinna-Cartan's characteristic function of a holomorphic map $f: \mathbf{C} \rightarrow \mathbf{P}^{n}(\mathbf{C})$, and by $\varsigma_{2}(f)$ the hyperorder of the holomorphic map $f$. Another holomorphic map $h$ is called to be "slowly" with respect to $f$ if $T_{h}(r)=o\left(T_{f}(r)\right)$. Denote by $N\left(r, \frac{1}{g}\right)$ the counting function of the zeros of an entire function $g$. The specific statement of the definitions can be seen in the next section.

Throughout this paper, we denote by $\mathcal{M}$ the set of all meromorphic functions on $\mathbf{C}$, by $\mathcal{P}_{c}$ the set of all meromorphic functions on $\mathbf{C}$ with period $c$, and by $\mathcal{P}_{c}^{\lambda}$ the set of all meromorphic functions with period $c$ on $\mathbf{C}$ and having the hyperorders strictly less than $\lambda$. Obviously, we have the relationship $\mathcal{M} \supset \mathcal{P}_{c} \supset \mathcal{P}_{c}^{\lambda}$.

The main purpose of this paper is to obtain the $c$-difference analogue of the second main theorem for holomorphic curves intersecting fixed or moving hypersurfaces in $N$ subgeneral position into $\mathbf{P}^{n}(\mathbf{C})$, which extends the difference analogue of the second main theorem in $[9,10]$ from hyperplanes to hypersurfaces and from general position to subgeneral position. We should note that this result is a difference version of the second main theorem due to [15].

Theorem 1.1. Let $f=\left[f_{0}: \cdots: f_{n}\right]: \mathbf{C} \rightarrow \mathbf{P}^{n}(\mathbf{C})$ be algebraically nondegenerate over $\mathcal{P}_{c}^{1}$ with hyperorder $\varsigma_{2}(f)<1$. Let $\left\{Q_{j}(z)\right\}_{j=1}^{q}$ be $c$-periodic slowly moving hypersurfaces of $\mathbf{P}^{n}(\mathbf{C})$ with respect to $f$ in (weakly) $N$-subgeneral position in $\mathbf{P}^{n}(\mathbf{C})$ with degree $d_{j}(1 \leq j \leq q)$. Let the least common multiple $d=l \mathrm{~cm}\left(d_{1}, \cdots, d_{q}\right)$ and $M=\left(\begin{array}{l}n+d \\ d\end{array}\right)-1$. Assume that $q>\frac{(M+1)(2 N-n+1)}{n+1}$. Then for any $\varepsilon>0$,

$$
\left(q-\frac{(M+1)(2 N-n+1)}{n+1}\right) T_{f}(r) \leq \sum_{j=1}^{q} \frac{1}{d_{j}} N\left(r, \frac{1}{Q_{j}(f)}\right)-\frac{n}{d N} N\left(r, \frac{1}{\tilde{C}(f)}\right)+o\left(T_{f}(r)\right)
$$


holds for all $r$ possibly outside of an exceptional set $E$ of finite logarithmic measure.

We note that the main result can be also extended to the case for meromorphic mappings from $\mathbf{C}^{m}$ into complex projective spaces $\mathbf{P}^{n}(\mathbf{C})$ by the standard process of averaging over the complex lines in the complex space $\mathbf{C}^{m}$, we refer to see two related references $[2,3]$ on this topic.

Remark 1.1. For the special case whenever hyperplanes $\left\{Q_{j}\right\}_{j=1}^{q}$ are located in general position (that is $d=d_{j}=1, M=N=n$ and $\tilde{C}(f)=C(f)$ ). Set $L=\frac{\prod_{j=1}^{q} Q_{j}(f)(z)}{C(f)}$. Clearly, Both $\prod_{j=1}^{q} Q_{j}(f)(z)$ and $C(f)$ are entire functions. By the Jensen's Formula (see in the next section), we have

$$
\begin{aligned}
& N\left(r, \frac{1}{L}\right)-N(r, L)=\int_{0}^{2 \pi} \log \left|L\left(r e^{\sqrt{-1} \theta}\right)\right| \frac{d \theta}{2 \pi}+O(1) \\
& =\sum_{j=1}^{q} \int_{0}^{2 \pi} \log \left|Q_{j}(f)\left(r e^{\sqrt{-1} \theta}\right)\right| \frac{d \theta}{2 \pi}-\int_{0}^{2 \pi} \log \left|C(f)\left(r e^{\sqrt{-1} \theta}\right)\right| \frac{d \theta}{2 \pi}+O(1) \\
& =\sum_{j=1}^{q} N\left(r, \frac{1}{Q_{j}(f)}\right)-N\left(r, \frac{1}{C(f)}\right)+O(1),
\end{aligned}
$$

and thus

$$
\begin{aligned}
\sum_{j=1}^{q} \frac{1}{d_{j}} N\left(r, \frac{1}{Q_{j}(f)}\right)-\frac{n}{d N} N\left(r, \frac{1}{\tilde{C}(f)}\right) & =\sum_{j=1}^{q} N\left(r, \frac{1}{Q_{j}(f)}\right)-N\left(r, \frac{1}{C(f)}\right) \\
& =N\left(r, \frac{1}{L}\right)-N(r, L)+O(1) .
\end{aligned}
$$

Hence according to Theorem 1.1, we have

$$
(q-(n+1)) T_{f}(r) \leq N\left(r, \frac{1}{L}\right)-N(r, L)+o\left(T_{f}(r)\right),
$$

which becomes the second main theorem for fixed hyperplanes in [9] and for $c$-periodic slowly moving hyperplanes targets in [10].

Definition 1.1. Let $k \in \mathbf{N}, c \in \mathbf{C} \backslash\{0\}$, and $a \in \mathbf{C}$. An $a$-point $z_{0}$ of an entire function $h(z)$ is said to be $k$-successive and $c$-separated, if the $k$ entire functions $h(z+\nu c)(\nu=1,2, \cdots, k)$ take the value $a$ at $z=z_{0}$ with multiplicity not less than that of $h(z)$ there. All the other $a$-points of $h(z)$ are called $k$-aperiodic of pace $c$. Denote by $\tilde{N}_{f}^{[k, c]}\left(r, \frac{1}{Q(f)(z)}\right)$ the counting function of $k$-aperiodic zeros of the entire function $Q(f)(z)$ of pace $c$ for the holomorphic curve $f$ intersecting a hypersurface $Q$.

In [10] Korhonen, Li and Tohge got a difference analogue of the truncated Second Main Theorem for moving hyperplanes in general position. Based on Theorem 1.1, we also obtain one difference analogue of the truncated Second Main Theorem for moving hypersurfaces in subgeneral position, which reduces to the result of Korhonen, Li and Tohge [10, Theorem 4.3] whenever $d_{j}=d=1$ and $M=N=n$.

Theorem 1.2. Let $f: \mathbf{C} \rightarrow \mathbf{P}^{n}(\mathbf{C})$ be algebraically nondegenerate over $\mathcal{P}_{c}^{1}$ with hyperorder $\varsigma_{2}(f)<1$. Let $\left\{Q_{j}(z)\right\}_{j=1}^{q}$ be c-periodic slowly moving hypersurfaces of $\mathbf{P}^{n}(\mathbf{C})$ with respect to $f$ in (weakly) $N$-subgeneral position with degree $d_{j}(1 \leq j \leq q)$. Let the least common multiple $d=\operatorname{lcm}\left(d_{1}, \cdots, d_{q}\right)$ and $M=\left(\begin{array}{l}n+d \\ d\end{array}\right)-1$. Assume that 
$q>\frac{(M+1)(2 N-n+1)}{n+1}$. Then for any $\varepsilon>0$, we have

$$
\begin{aligned}
& \left(q-\frac{(M+1)(2 N-n+1)}{n+1}\right) T_{f}(r) \\
& \leq \sum_{j=1}^{q} \frac{1}{d_{j}} \tilde{N}_{f}^{[M, c]}\left(r, \frac{1}{Q_{j}(f)(z)}\right)+\frac{N-n}{d N} N\left(r, \frac{1}{\tilde{C}(f)}\right)+o\left(T_{f}(r)\right),
\end{aligned}
$$

for all $r$ outside of a set $E$ with finite logarithmic measure.

The remainder of this paper is organized in the following way. In Section 2, some notations and basic results of Nevanlinna theory for hypersurfaces are introduced briefly. In Section 3, we give some lemmas for the proofs of our main theorems. In Section 4, the proof of Theorem 1.1 is shown. Finally, Theorem 1.2 is proved in the last section.

\section{Preliminaries}

Let $\mathbf{P}^{n}(\mathbf{C})=\mathbf{C}^{n+1} \backslash\{0\} / \sim$, where $\left(a_{0}, a_{1}, \ldots, a_{n}\right) \sim\left(b_{0}, b_{1}, \ldots, b_{n}\right)$ if and only if $\left(a_{0}, a_{1}, \ldots, a_{n}\right)=\lambda\left(b_{0}, b_{1}, \ldots, b_{n}\right)$ for some $\lambda \in \mathbf{C}$. We denote by $\left[a_{0}: \cdots: a_{n}\right]$ the equivalent class of $\left(a_{0}, a_{1}, \ldots, a_{n}\right)$. Let

$$
f=\left[f_{0}: \cdots: f_{n}\right]: \mathbf{C} \rightarrow \mathbf{P}^{n}(\mathbf{C})
$$

be a holomorphic map where $f_{0}, \ldots, f_{n}$ are entire functions and without common zeros. Usually, $f$ is called holomorphic curve. Denote by $\mathbf{f}=\left(f_{0}, \ldots, f_{n}\right)$. Then $\mathbf{f}$ is called a reduced representation of $f$. We recall the Nevanlinna-Cartan's characteristic function $T_{f}(r)$ of $f$ defined as

$$
T_{f}(r)=\int_{0}^{2 \pi} \log \left\|f\left(r e^{\sqrt{-1} \phi}\right)\right\| \frac{d \phi}{2 \pi}+\log \|f(0)\|,
$$

where $\|f\|=\max _{0 \leq j \leq n}\left\{\left|f_{j}\right|\right\}$. Note that the Cartan's characteristic function does not depend on the choice of the reduced representation. The order and hyperorder of $f$ are defined as

$$
\varsigma(f)=\lim \sup _{r \rightarrow \infty} \frac{\log ^{+} T_{f}(r)}{\log r}
$$

and

$$
\varsigma_{2}(f)=\lim \sup _{r \rightarrow \infty} \frac{\log ^{+} \log ^{+} T_{f}(r)}{\log r},
$$

respectively, where $\log ^{+} x=\max \{\log x, 0\}$ for $x>0$.

For a meromorphic function $g$ on $\mathbf{C}$, and let $a \in \mathbf{C}$ and $g(0) \neq a, \infty$, we have the first main theorem

$$
T_{\frac{1}{g-a}}(r)=T_{g}(r)+O(1) .
$$

Denote by $n(r, g)$ the number of poles of $g$ in the disc $|z|<r$ (counting multiplicity), and define the counting function of poles of $g$ by

$$
N(r, g)=\int_{0}^{r}[n(r, g)-n(0, g)] \frac{d t}{t}+n(0, g) \log r .
$$

Then the first main theorem implies the Jensen's Formula

$$
N(r, 1 / g)-N(r, g)=\frac{1}{2 \pi} \int_{0}^{2 \pi} \log \left|g\left(r e^{\sqrt{-1} \theta}\right)\right| d \theta+O(1) .
$$


A hypersurface $Q$ with degree $d$ in $\mathbf{P}^{n}(\mathbf{C})$ is given by

$$
Q=\left\{\left[x_{0}: \cdots: x_{n}\right]: \sum_{I \in \mathcal{J}_{d}} a_{I} x^{I}=0\right\}
$$

where $\mathcal{J}_{d}=\left\{\left(i_{0}, \cdots, i_{n}\right) \in \mathbf{N}_{0}^{n+1}: i_{0}+\cdots+i_{n}=d\right\}, I=\left(i_{0}, \cdots, i_{n}\right) \in \mathcal{J}_{d}, x^{I}=$ $x_{0}^{i_{0}} \cdots x_{n}^{i_{n}}$ and $\left(x_{0}: \cdots: x_{n}\right)$ is homogeneous coordinates of $\mathbf{P}^{n}(\mathbf{C})$. Denote by $H_{d}$ the vector set of all homogeneous polynomials in $\mathbf{C}\left[x_{0}, \cdots, x_{n}\right]$ of degree $d$. Then clearly, we have $\operatorname{dim} H_{d}=M+1$, where $M=\left(\begin{array}{c}n+d \\ d\end{array}\right)-1$. Denote by $D$ the homogeneous polynomial $\sum_{I \in \mathcal{J}_{d}} a_{I} x^{I}$ associate with the hypersurface $Q$. Thus the hypersurface $Q$ defines a vector, say $a=\left(a_{0}, \ldots, a_{M}\right)$ in $\mathbf{C}^{M+1}$. If $d$ is 1 , then the hypersurface reduces to one hyperplane.

If all the above $a_{I}$ are meromorphic functions on $\mathbf{C}$ in the definition of the hypersurface $Q$, then we say that the $Q$ is a moving hypersurface. If all the meromorphic functions $a_{I}$ are $c$-peroidic, then we say that the $Q$ is $c$-periodic moving hypersurface. Furthermore, the $Q$ is said to be slowly moving hypersurface with respect to the holomorphic curve $f$ if all $a_{I}$ satisfy $T_{a_{I}}(r)=o\left(T_{f}\right)(r)$. Let $D$ be the homogeneous polynomial (form) of degree $d$ defining the moving hypersurface $Q$. For a holomorphic curve $f=\left[f_{0}: \cdots: f_{n}\right]: \mathbf{C} \rightarrow \mathbf{P}^{n}(\mathbf{C})$ and the hypersurface $Q$, set

$$
Q(f)(z):=D \circ f(z)=\sum_{I \in \mathcal{J}_{d}} a_{I} f^{I}
$$

where $f^{I}=f_{0}^{i_{0}} \cdots f_{n}^{i_{n}}$. We recall the proximity function of $f$ intersecting $Q$ defined as

$$
m_{f}(r, Q)=\int_{0}^{2 \pi} \log \frac{\max _{0 \leq j \leq n}\left\{\left|f_{j}\left(r e^{\sqrt{-1} \theta}\right)\right|^{d}\right\} \max _{0 \leq j \leq n}\left\{\left|a_{i_{j}}\left(r e^{\sqrt{-1} \theta}\right)\right|^{d}\right\}}{\left|Q(f)\left(r e^{\sqrt{-1} \theta}\right)\right|} \frac{d \theta}{2 \pi} .
$$

Throughout this paper, we usually assume that $f(\mathbf{C}) \not \subset Q$ if without special statement. Then we have the first main theorem as follows:

$$
m_{f}(r, Q)+N\left(r, \frac{1}{Q(f)}\right)=d T_{f}(r)+O(1) .
$$

Now let $\left\{Q_{i}(z)\right\}_{i}^{q}$ be (moving) hypersurfaces of $\mathbf{P}^{n}(\mathbf{C})$. Set $N \geq n$ and $q \geq N+1$. We say that the family of the hypersurfaces $\left\{Q_{j}(z)\right\}_{j=1}^{q}$ are in (weakly) $N$-subgeneral position in $\mathbf{P}^{n}(\mathbf{C})$ if for any subset $R \subset\{1, \cdots, q\}$ with the cardinality $\sharp R=N+1$ (and for any $z \in \mathbf{C}$ ), we have

$$
\bigcap_{j \in R} Q_{j}(z)=\emptyset
$$

That is, any $N+1$ homogeneous polynomials (form) of $\left\{D_{j}(z)\right\}_{j=1}^{q}$ which associate with the hypersurfaces $\left\{Q_{j}(z)\right\}_{j=1}^{q}$ are linearly independent over $\mathbf{C}$. Whenever $N=$ $n$, we say that the hypersurfaces $\left\{Q_{i}(z)\right\}_{i}^{q}$ are in (weakly) general position in $\mathbf{P}^{n}(\mathbf{C})$.

\section{Some lemmas}

Before giving the proofs of our main results, we need some lemmas as follows. The first one is the difference analogue of the logarithmic derivative lemma for meromorphic functions due to Halburd, Korhonen and Tohge. We note that Chiang and Feng [6] also obtained one version of the difference analogue of the logarithmic derivative lemma for meromorphic functions with finite order but without the possible exceptional set. 
Lemma 3.1. $[8,9]$ Let $f$ be a nonconstant meromorphic function, for all $\varepsilon>0$ and $c \in \mathbf{C} \backslash\{0\}$. If $f$ is of finite order, then there exists a set $E=E(f, \varepsilon)$ satisfying

$$
\limsup _{r \rightarrow \infty} \frac{\int_{E \cap[1, r)} \frac{d t}{t}}{\log r} \leq \varepsilon
$$

i.e. of logarithmic density at most $\varepsilon$, such that

$$
m\left(r, \frac{f(z+c)}{f(z)}\right):=\int_{0}^{2 \pi} \log ^{+}\left|\frac{f\left(r e^{\sqrt{-1} \theta}+c\right)}{f\left(r e^{\sqrt{-1} \theta}\right)}\right| \frac{d \theta}{2 \pi}=O\left(\frac{\log r}{r} T_{f}(r)\right)
$$

for all $r$ outside the set $E$. If $\varsigma_{2}(f)=\varsigma_{2}<1$ and $\varepsilon>0$, then

$$
m\left(r, \frac{f(z+c)}{f(z)}\right)=o\left(\frac{T_{f}(r)}{r^{1-\varsigma_{2}-\varepsilon}}\right)
$$

for all $r$ outside of a set of finite logarithmic measure.

Since

$$
\frac{f(z)}{f(z+c)}=\frac{f[(z+c)-c]}{f(z+c)}, \quad \frac{\bar{f}^{[k]}}{f(z)}=\frac{\bar{f}^{[k]}}{\bar{f}^{[k-1]}} \cdot \frac{\bar{f}^{[k-1]}}{\bar{f}^{[k-2]}} \cdots \frac{\bar{f}}{f(z)},
$$

for $k \in \mathbf{N}$, it follows immediately from Lemma 3.1 that for a meromorphic function $f$ of hyperorder $\varsigma_{2}(f)<1$,

$$
m\left(r, \frac{\bar{f}^{[k]}}{f(z)}\right)=o\left(\frac{T_{f}(r)}{r^{1-\varsigma_{2}(f)-\varepsilon}}\right)
$$

holds for any $\varepsilon>0$ and for all $r$ outside of a set of finite logarithmic measure.

It was mentioned without the proof $[18$, Remark 2.6] that holomorphic functions $g_{0}, \ldots, g_{n}$ on $\mathbf{C}$ are linearly dependent over $\mathcal{P}_{c}$ if and only if their Casorati determinant $C\left(g_{0}, \ldots, g_{n}\right)$ vanishes identically. The proof of this fact can be seen in the proof of [9, Lemma 3.2] which in fact, is a more accurate result by considering the growth order of functions. Here we extend their results to algebraically independent over $\mathcal{P}_{c}^{\lambda}$.

Lemma 3.2. Let $c \in \mathbf{C} \backslash\{0\}$. Set $M=\left(\begin{array}{c}n+d \\ d\end{array}\right)-1, \mathcal{J}_{d}=\left\{\left(i_{0}, \cdots, i_{n}\right) \in \mathbf{N}_{0}^{n+1}: i_{0}+\right.$ $\left.\cdots+i_{n}=d\right\}, I_{j} \in \mathcal{J}_{d}$ for all $j \in\{0,1, \ldots, M\}$. Then the holomorphic curve $f=\left[f_{0}, \cdots, f_{n}\right]: \mathbf{C} \rightarrow \mathbf{P}^{n}(\mathbf{C})$ with hyperorder strictly less than $\lambda$ satisfies $\tilde{C}(f)=$ $C\left(f^{I_{0}}, \ldots, f^{I_{M}}\right) \not \equiv 0$ if and only if the entire functions $f_{0}, \ldots, f_{n}$ are algebraically nondegenerate over the field $\mathcal{P}_{c}^{\lambda}$.

Proof. Set $g_{j}=f^{I_{j}}(j=0,1, \ldots, M)$. Then $g=\left[g_{0}: g_{1}: \cdots: g_{M}\right]$ is a holomorphic map from $\mathbf{C}$ into $\mathbf{P}^{M}(\mathbf{C})$. According to the definition of the NevanlinnaCartan's characteristic function,

$$
\begin{aligned}
T_{g}(r) & =\int_{0}^{2 \pi} \log \max _{0 \leq j \leq M}\left\{\left|f^{I_{j}}\left(r e^{\sqrt{-1} \theta}\right)\right|\right\} \frac{d \theta}{2 \pi}+O(1) \\
& =\int_{0}^{2 \pi} \log \max _{0 \leq j \leq M}\left\{\left|g_{j}\left(r e^{\sqrt{-1} \theta}\right)\right|\right\} \frac{d \theta}{2 \pi}+O(1) \\
& \leq \int_{0}^{2 \pi} \log \max _{0 \leq j \leq n}\left\{\left|f_{j}\left(r e^{\sqrt{-1} \theta}\right)\right|^{d}\right\} \frac{d \theta}{2 \pi}+O(1) \\
& =d T_{f}(r)+O(1) .
\end{aligned}
$$


Hence, by the definition of the hyperorder, we have $\varsigma_{2}(g) \leq \varsigma_{2}(f)<\lambda$. Note that $f$ is algebraically nondegenerate (over the field $\mathcal{P}_{c}^{\lambda}$ ) if and only if $g$ is linearly nondegenerate (over the field $\mathcal{P}_{c}^{\lambda}$ ). Then by [9, Lemma 3.2] we get that $g$ is linearly nondegenerate over the field $\mathcal{P}_{c}^{\lambda}$ if and only if $C(g)=C\left(g_{0}, \ldots, g_{M}\right) \not \equiv 0$, and thus we complete the proof of the lemma.

The Nochka's method $[12,13]$ plays the key role in extending the Cartan's second main theorem from general position to the subgeneral position for hyperplanes. In $[1,15]$, An, Quang and Thai extended the Nochka's method to the case for hypersurfaces in subgeneral position. Here we extend their results to the case for moving hypersurfaces in subgeneral position. The $\omega_{j}$ and $\tilde{\omega}$ are called the Nochka weights and the Nochka constant, respectively.

Lemma 3.3. Let $Q_{1}(z), \cdots, Q_{q}(z)$ be $q(q>2 N-n+1)$ moving hypersurfaces with the common degree $d$ and in (weakly) $N$-subgeneral position of $\mathbf{P}^{n}(\mathbf{C})$. Then there are positive rational constants $\omega_{i}(1 \leq i \leq q)$ satisfying the following:

i) $0<\omega_{i} \leq 1$ for all $i \in\{1, \cdots, q\}$;

ii) Setting $\tilde{\omega}=\max _{1 \leq j \leq q} \omega_{j}$, one gets

$$
\sum_{j=1}^{q} \omega_{j}=\tilde{\omega}(q-2 N+n-1)+n+1
$$

iii) $\frac{n+1}{2 N-n+1} \leq \tilde{\omega} \leq \frac{n}{N}$;

iv) For $R \subset\{1, \cdots, q\}$ with $\sharp R=N+1$, then $\sum_{j \in R} \omega_{j} \leq \operatorname{rank}\left\{Q_{j}\right\}_{j \in R}+1$;

v) Let $E_{j} \geq 1(1 \leq j \leq q)$ be arbitrarily given numbers. For $R \subset\{1, \cdots, q\}$ with $\sharp R=N+1$, there is a subset $R^{0} \subset R$ such that $\# R^{0}=\operatorname{rank}\left\{Q_{i}\right\}_{i \in R^{0}}=n+1$ and

$$
\prod_{i \in R} E_{i}^{\omega_{i}} \leq \prod_{i \in R^{0}} E_{i}
$$

Proof. For any fixed $z, Q_{1}(z), \ldots, Q_{q}(z)$ can be seen fixed hypersurfaces in $N$ subgeneral position. Then by $[1,15]$, the conclusion of the lemma is true. So the lemma is proved.

Let $\left\{Q_{j}(z)\right\}_{j=1}^{q}$ be $q$ (moving) hypersurfaces in $\mathbf{P}^{n}(\mathbf{C})$ of the common degree d. Assume that each $Q_{j}(z)$ is defined by a homogeneous polynomial $D_{j}(z)$ in $\mathbf{C}\left[x_{0}, \cdots, x_{n}\right]$. We regard $\mathbf{C}\left[x_{0}, \cdots, x_{n}\right]$ as a complex vector space and define

$$
\operatorname{rank}\left\{Q_{j}(z)\right\}_{j \in K}=\operatorname{rank}\left\{D_{j}(z)\right\}_{j \in K}
$$

for every subset $K \subset\{1,2, \cdots, q\}$. It is easy that

$$
\operatorname{rank}\left\{Q_{j}(z)\right\}_{j \in K}=\operatorname{rank}\left\{D_{j}(z)\right\}_{j \in K} \geq n+1-\operatorname{dim}\left(\bigcap_{j \in K} Q_{j}(z)\right) .
$$

The following lemma will play the important role in the proofs of our main theorems. We point out that this comes from [15, Lemma 4.2], but here we give another proof and also give a way how to take the hypersurfaces $\left\{T_{j}\right\}_{j=1}^{M-k}$.

Lemma 3.4. Let $\left\{Q_{j}(z)\right\}_{j=1}^{q}$ be a set of $q$ moving hypersurfaces in $\mathbf{P}^{n}(\mathbf{C})$ of the common degree $d$. Then for some subset $R \subset\{1, \cdots, q\}$ with $\sharp R=\operatorname{rank}\left\{Q_{j}(z)\right\}_{j \in R}=$ $k+1$, there exist $(M-k)$ moving hypersurfaces $\left\{T_{j}(z)\right\}_{j=1}^{M-k}$ of degree $d$ in $\mathbf{P}^{n}(\mathbf{C})$ 
whose coefficients are chosen from coefficients of $\left\{Q_{j}(z)\right\}_{j \in R}$, such that the rank of $\left\{Q_{j}(z)\right\}_{j \in R} \cup\left\{T_{j}(z)\right\}_{j=1}^{M-k}$ is $M+1$.

Proof. Let $\left\{Q_{j}(z)\right\}_{j=1}^{q}$ be moving hypersurfaces which vector functions are $a_{j}(z)=$ $\left(a_{j 0}(z), a_{j 1}(z), \cdots, a_{j M}(z)\right)$. Since $\operatorname{rank}\left\{Q_{j}(z)\right\}_{j \in R}=k+1=\sharp R$, without loss of generality, we may assume them to regard as $\left\{Q_{j}\right\}_{j=1}^{k+1}$. Denote by $H_{1}(z)$ the $(k+1) \times(M+1)$ matrix consisting of the corresponding vector functions of moving hypersurfaces $Q_{1}(z), \cdots, Q_{k+1}(z)$. We will get a $(M+1) \times(M+1)$ function matrix $H(z)$ as follows:

$$
H(z)=\left(\begin{array}{c}
H_{1}(z) \\
O
\end{array}\right)=\left(\begin{array}{cccc}
a_{10}(z) & a_{11}(z) & \cdots & a_{1 M}(z) \\
a_{20}(z) & a_{11}(z) & \cdots & a_{2 M}(z) \\
\vdots & \vdots & \vdots & \vdots \\
a_{(k+1) 0}(z) & a_{(k+1) 1}(z) & \cdots & a_{(k+1) M}(z) \\
0 & 0 & \cdots & 0 \\
\vdots & \vdots & \vdots & \vdots \\
0 & 0 & \cdots & 0
\end{array}\right)_{(M+1) \times(M+1)},
$$

where $O$ is a $(M+1) \times(M-k)$ zero-matrix.

We will decompose $H(z)$ into four matrixes as a $(k+1) \times(k+1)$ matrix $H_{2}(z)$, a $(k+1) \times(M-k)$ matrix $H_{3}(z)$, a $(M-k) \times(k+1)$ zero-matrix $O_{1}$ and a $(M-k) \times(M-k)$ zero-matrix $O_{2}$ such that

$$
H(z)=\left(\begin{array}{cc}
H_{2}(z) & H_{3}(z) \\
O_{1} & O_{2}
\end{array}\right)
$$

where the determinant of $H_{2}(z)$ is not 0 , since $\operatorname{rank}\left\{Q_{j}(z)\right\}_{j=0}^{k}=k+1$. Set

$$
G(z):=\left(\begin{array}{cc}
E_{1} & -\left(H_{2}(z)\right)^{-1} \cdot H_{3}(z) \\
O_{1} & E_{2}
\end{array}\right)
$$

where $E_{1}, E_{2}$ are the $(k+1) \times(k+1)$ identity matrix and the $(M-k) \times(M-k)$ identity matrix respectively. Then we have

$$
H(z) G(z)=\left(\begin{array}{cc}
H_{2}(z) & O_{3} \\
O_{1} & O_{2}
\end{array}\right)
$$

where $O_{3}$ is a $(k+1) \times(k+1)$ zero-matrix. Therefore, there exists at least one $a_{i j}(z) \neq 0$ in $H(z)$. Since $\operatorname{det}\left(H_{2}(z)\right) \neq 0$, we may assume $a_{10}(z) \neq 0$. Set

$$
F(z)=\left(\begin{array}{cc}
H_{2}(z) & O_{3} \\
O_{1} & a_{10}(z) E_{2}
\end{array}\right)_{(M+1) \times(M+1)} .
$$

Obviously, the rank of $F(z)$ is $M+1$. So we have

$$
F(z)(G(z))^{-1}=\left(\begin{array}{cc}
H_{2}(z) & H_{3}(z) \\
O_{1} & a_{10}(z) E_{2}
\end{array}\right)
$$

this implies that the rank of $F(z)(G(z))^{-1}$ is $M+1$. Therefore, we can take these moving hypersurfaces $\left\{T_{i}(z)\right\}_{i=1}^{M-k}$ associated with corresponding vector matrix defined by $\left(O_{1}, a_{10}(z) E\right)$, which satisfy $\operatorname{rank}\left\{\left\{Q_{i}(z)\right\}_{i \in R} \cup\left\{T_{i}(z)\right\}_{i=1}^{M-k}\right\}=M+1$.

Finally, we show a result which is a different version of [7, Lemma 2.2]. 
Lemma 3.5. Let $Q_{1}(z), \cdots, Q_{q}(z)$ be $q$ moving hypersurfaces with the common degree $d$ in $\mathcal{K}_{f}\left[x_{0}, \cdots, x_{n}\right]$. Then there exists a function $h_{1} \in \mathcal{C}_{f}$ such that,

$$
\max _{j \in\{1, \cdots, q\}}\left\{\left|Q_{j}(f)(z)\right|\right\} \leq h_{1} \cdot\|f\|^{d} .
$$

If, moreover, for any set $R \subset\{1, \ldots, q\}$ such that $\operatorname{rank}(R)=k+1=\sharp R$, then there exist $M-k$ (moving) hypersurfaces $\left\{T_{i}(z)\right\}_{i=1}^{M-k}$ satisfying the condition of Lemma 3.4 and nonzero functions $h_{2}, h_{3} \in \mathcal{C}_{f}$ such that,

$$
h_{2} \cdot\|f\|^{d} \leq \max _{j \in R}\left\{\left|Q_{j}(f)(z)\right|\right\}
$$

and

$$
h_{3} \cdot\|f\|^{d} \leq \max _{j \in\{1, \cdots, M-k\}}\left\{\left|T_{j}(f)(z)\right|\right\},
$$

where $\mathcal{K}_{f}$ is the set of all "small" (with respect to $f$ ) entire functions on $\mathbf{C}$, and $\mathcal{C}_{f}$ is the set of all non-negative functions $h: \mathbf{C} \rightarrow(0,+\infty]$ such that

$$
\int_{0}^{2 \pi} \log h\left(r e^{\sqrt{-1} \phi}\right) \frac{d \phi}{2 \pi}=o\left(T_{f}(r)\right)
$$

Proof. Let $\operatorname{rank}\left\{Q_{j}(z)\right\}_{j \in R}=k+1$, by Lemma 3.4, we can find $M-k$ (moving) hypersurfaces $\left\{T_{i}(z)\right\}$ of degree $d$ such that $\operatorname{rank}\left\{\left\{Q_{j}(z)\right\}_{j \in R} \cup\left\{T_{i}(z)\right\}_{i=1}^{M-k}\right\}=M+$ 1. Hence the $M+1$ moving hypersurfaces $\left\{Q_{j}(z)\right\}_{j=1}^{k+1} \cup\left\{T_{i}(z)\right\}_{i=1}^{M-k}$ are admissible (namely, linearly independent). Then by [7, Lemma 2.2], we get that the lemma is proved.

\section{Proof of Theorem 1.1}

Firstly, we may assume that the homogeneous moving hypersurfaces $\left\{Q_{j}\right\}_{j=1}^{q}$ are homogeneous polynomials of degree $d$ in $N$-subgeneral position in $\mathbf{P}^{n}(\mathbf{C})$ in $\mathbf{C}\left[x_{0}, \cdots, x_{n}\right]$. Set $Q=\{1, \ldots, q\}$ and $M=\left(\begin{array}{l}n+d \\ d\end{array}\right)-1$. Let $\left\{e_{i}\right\}_{i=0}^{M}$ be a standard basis of the ring of all homogeneous polynomials of degree $d$ in $\mathbf{C}\left[x_{0}, \cdots, x_{n}\right]$. Since $f$ is an algebraically nondegenerate holomorphic mapping of $\mathbf{C}$ into $\mathbf{P}^{n}(\mathbf{C})$ over $\mathcal{P}_{c}^{1}$, it implies that $\left\{e_{i}(f) ; 0 \leq i \leq M\right\}$ is linearly independent over $\mathcal{P}_{c}^{1}$. Then by Lemma 3.2 , we get $\tilde{C}(f) \not \equiv 0$. Without loss of generality, we may assume

$$
B_{0}(f) \equiv\left|\begin{array}{cccc}
e_{0}(f) & e_{1}(f) & \cdots & e_{M}(f) \\
e_{0}(\bar{f}) & e_{1}(\bar{f}) & \cdots & e_{M}(\bar{f}) \\
\vdots & \vdots & \vdots & \vdots \\
e_{0}\left(\bar{f}^{[M]}\right) & e_{1}\left(\bar{f}^{[M]}\right) & \cdots & e_{M}\left(\bar{f}^{[M]}\right)
\end{array}\right|=\tilde{C}(f) .
$$

For each $R^{0}=\left\{r_{1}^{0}, \cdots, r_{n+1}^{0}\right\} \subset\{1, \cdots, q\}$ with $\operatorname{rank}\left\{Q_{i}\right\}_{i \in R^{0}}=\sharp R^{0}=n+1$. Then by Lemma 3.4, we can choose $\left\{T_{j}(z)\right\}_{j=1}^{M-n}$ be $M-n$ hypersurfaces of degree $d$ in $\mathbf{P}^{n}(\mathbf{C})$ whose coefficients are chosen from coefficients of $\left\{Q_{j}(z)\right\}_{j \in R^{0}}$, such that $\operatorname{rank}\left\{\left\{Q_{j}(z)\right\}_{j \in R^{0}} \cup\left\{T_{j}(z)\right\}_{j=1}^{M-n}\right\}=M+1$. Set

$$
\begin{aligned}
B(f) & \equiv\left|\begin{array}{cccccc}
Q_{r_{1}^{0}}(f) & \cdots & Q_{r_{n+1}^{0}}(f) & T_{1}(f) & \cdots & T_{M-n}(f) \\
Q_{r_{1}^{0}}(\bar{f}) & \cdots & Q_{r_{n+1}^{0}}(\bar{f}) & T_{1}(\bar{f}) & \cdots & T_{M-n}(\bar{f}) \\
\vdots & \vdots & \vdots & \vdots & \vdots & \vdots \\
Q_{r_{1}^{0}}\left(\bar{f}^{[M]}\right) & \cdots & Q_{r_{n+1}^{0}}\left(\bar{f}^{[M]}\right) & T_{1}\left(\bar{f}^{[M]}\right) & \cdots & T_{M-n}\left(\bar{f}^{[M]}\right)
\end{array}\right| \\
& =|(e(z) \times \tilde{C}(f))|,
\end{aligned}
$$


where $e(z)$ is the coefficients matrix of $\left\{Q_{i}(z)\right\}_{i \in R^{0}} \cup\left\{T_{i}(z)\right\}_{i=1}^{M-n}$ under the basis $\left\{e_{i}\right\}_{i=0}^{M}$.

By Lemma 3.5, there exists a function $\beta$ which satisfies the following condition:

$$
\left\{\frac{\beta\|f(z)\|^{d}}{\left|Q_{i}(f)\right|}\right\}_{i=1}^{q} \geq 1, \quad \int_{0}^{2 \pi} \log \beta\left(r e^{\sqrt{-1} \phi}\right) d \phi=o\left(T_{f}(r)\right) .
$$

For any given $z$, we can rearrange the $\left\{Q_{j}(f)(z)\right\}_{j=1}^{q}$ according to the increasing order of their modulus as follows

$$
\left|Q_{r_{1}}(f)(z)\right| \leq\left|Q_{r_{2}}(f)(z)\right| \leq \cdots \leq\left|Q_{r_{q}}(f)(z)\right| .
$$

Now choose $R=\left\{r_{1}, \cdots, r_{N+1}\right\} \subset Q$ with $\sharp R=N+1$, by Lemma $3.3 \mathrm{v}$ ) we get that there exists one subset $R^{0}=\left\{r_{1}^{0}, \cdots, r_{n+1}^{0}\right\} \subset R \subset Q$ with $\operatorname{rank}\left\{Q_{i}\right\}_{i \in R^{0}}=\sharp R^{0}=$ $n+1$, such that

$$
\prod_{i \in R}\left(\frac{\beta\|f(z)\|^{d}}{\left|Q_{i}(f)\right|}\right)^{\omega_{i}} \leq \prod_{i \in R^{0}} \frac{\beta\|f(z)\|^{d}}{\left|Q_{i}(f)\right|},
$$

where $\left\{\omega_{i}\right\}_{i=1}^{q}$ are the Nochka weights. Let $S=Q \backslash R$. Then by Lemma 3.5, it follows that for the given $z$,

$$
h_{1}(z)\|f(z)\|^{d} \leq \max _{i \in R}\left|Q_{i}(f)(z)\right| \leq \min _{j \in S}\left\{\left|Q_{j}(f)(z)\right|\right\},
$$

and by the way of taking the $\left\{T_{i}(f)\right\}_{i=1}^{M-n}$ in Lemma 3.4 and by Lemma 3.5 it gives

$$
h_{2}(z)\|f(z)\|^{d} \leq \max _{\{1, \cdots, M-n\}}\left|T_{i}(f)(z)\right| \leq h_{3}(z)\|f(z)\|^{d},
$$

where hypersurfaces $T_{i}(f)(z)$ are of degree $d$ in $\mathbf{P}^{n}(\mathbf{C})$ whose coefficients chosen from coefficients of $\left\{Q_{j}(z)\right\}_{j \in R^{0}}$ such that rank $\left\{\left\{Q_{j}(z)\right\}_{j \in R^{0}} \cup\left\{T_{j}(z)\right\}_{j=1}^{M-n}\right\}=M+1$, and $h_{1}, h_{2}, h_{3} \in \mathcal{C}_{f}$. Then, we can get

$$
\begin{aligned}
& \frac{\|f(z)\|^{d\left(\sum_{i=1}^{q} \omega_{i}\right)}|B(f)|}{\left|Q_{1} \circ f(z)\right|^{\omega_{1}} \cdots\left|Q_{q} \circ f(z)\right|^{\omega_{q}}}=\frac{\|f(z)\|^{d\left(\sum_{i \in Q} \omega_{i}\right)}|B(f)|}{\prod_{i \in Q}\left|Q_{i}(f(z))\right|^{\omega_{i}}} \\
& =\frac{\|f(z)\|^{d\left(\sum_{i \in R} \omega_{i}\right)}\|f(z)\|^{d\left(\sum_{i \in S} \omega_{i}\right)}|B(f)|}{\left.\prod_{i \in R}\left|Q_{i}(f(z))\right| \omega_{i}\left|\prod_{i \in S}\right| Q_{i}(f(z))\right|^{\omega_{i}}} \\
& =\frac{|B(f)|}{\beta^{\sum_{i \in R} \omega_{i}}} \prod_{i \in R}\left(\frac{\beta\|f(z)\|^{d}}{\left|Q_{i}(f(z))\right|}\right)^{\omega_{i}} \prod_{i \in S}\left(\frac{\|f(z)\|^{d}}{\left|Q_{i}(f(z))\right|}\right)^{\omega_{i}} \\
& \leq \frac{|B(f)|}{h_{1}(z)^{\sum_{i \in S} \omega_{i}} \beta^{\sum_{i \in R} \omega_{i}}} \prod_{i \in R}\left(\frac{\beta\|f(z)\|^{d}}{\left|Q_{i}(f(z))\right|}\right)^{\omega_{i}},
\end{aligned}
$$

this together with (1) implies that

$$
\begin{aligned}
& \frac{\|f(z)\|^{d\left(\sum_{i=1}^{q} \omega_{i}\right)}|B(f)|}{\left|Q_{1} \circ f(z)\right|^{\omega_{1}} \cdots\left|Q_{q} \circ f(z)\right|^{\omega_{q}}} \leq \frac{|e(z)| \cdot\left|B_{0}(f)\right|}{h_{1}(z)^{\sum_{i \in S} \omega_{i}} \beta^{\sum_{i \in R} \omega_{i}}}\left(\frac{\beta^{n+1}\|f(z)\|^{d(n+1)}}{\prod_{i \in R^{0}}\left|Q_{i}(f(z))\right|}\right) \\
& \leq \frac{\beta^{n+1}|e(z)| \cdot\left|B_{0}(f)\right| \cdot\|f(z)\|^{d(M+1)}}{h_{1}(z)^{\sum_{i \in S} \omega_{i}} \beta^{\sum_{i \in R} \omega_{i}} h_{3}(z)^{M-n} \prod_{i \in R^{0}}\left|Q_{i}(f(z))\right| \prod_{i=1}^{M-n}\left|T_{i}(f(z))\right|}
\end{aligned}
$$




$$
\begin{aligned}
= & \frac{\beta^{n+1}|e(z)| \cdot\left|B_{0}(f)\right| \cdot\|f(z)\|^{d(M+1)}}{h_{1}(z)^{\sum_{i \in S} \omega_{i}} \beta^{\sum_{i \in R} \omega_{i}} h_{3}(z)^{M-n} \prod_{r_{i}^{0} \in R^{0}}\left|Q_{r_{i}^{0}}\left(\bar{f}^{[i-1]}(z)\right)\right| \prod_{i=1}^{M-n}\left|T_{i}\left(\bar{f}^{[n+i]}(z)\right)\right|} \\
& \cdot \frac{\prod_{r_{i}^{0} \in R^{0}}\left|Q_{r_{i}^{0}}\left(\bar{f}^{[i-1]}(z)\right)\right| \prod_{i=1}^{M-n}\left|T_{i}\left(\bar{f}^{[n+i]}(z)\right)\right|}{\prod_{r_{i}^{0} \in R^{0}}\left|Q_{r_{i}^{0}}(f(z))\right| \prod_{i=1}^{M-n}\left|T_{i}(f(z))\right|}
\end{aligned}
$$

for the given $z$. Set

$$
\begin{aligned}
S_{R} & =\frac{\beta^{n+1}|e(z)| \cdot\left|B_{0}(f)\right|}{h_{1}(z)^{\sum_{i \in S} \omega_{i}} \beta_{i \in R} \omega_{i} h_{3}(z)^{M-n} \prod_{r_{i}^{0} \in R^{0}}\left|Q_{r_{i}^{0}}\left(\bar{f}^{[i-1]}(z)\right)\right| \prod_{i=1}^{M-n}\left|T_{i}\left(\bar{f}^{[n+i]}(z)\right)\right|} \\
& =\frac{K \cdot|e(z)| \cdot\left|B_{0}(f)\right|}{\prod_{r_{i}^{0} \in R^{0}}\left|Q_{r_{i}^{0}}\left(\bar{f}^{[i-1]}(z)\right)\right| \prod_{i=1}^{M-n}\left|T_{i}\left(\bar{f}^{[n+i]}(z)\right)\right|}
\end{aligned}
$$

where $K \in \mathcal{C}_{f}$ and

$$
A=\frac{\left.\prod_{r_{i}^{0} \in R^{0}} \mid Q_{r_{i}^{0}} \bar{f}^{[i-1]}(z)\right)\left|\prod_{i=1}^{M-n}\right| T_{i}\left(\bar{f}^{[n+i]}(z)\right) \mid}{\prod_{r_{i}^{0} \in R^{0}}\left|Q_{r_{i}^{0}}(f(z))\right| \prod_{i=1}^{M-n}\left|T_{i}(f(z))\right|} .
$$

By the above inequality, we get that

$$
\log \left(\frac{\|f(z)\|^{d\left(\sum_{i=1}^{q} \omega_{i}\right)}|B(f)|}{\left|Q_{1}(f(z))\right|^{\omega_{1}} \cdots\left|Q_{q}(f(z))\right|^{\omega_{q}}}\right) \leq \log \left(\|f(z)\|^{d(M+1)}\right)+\log ^{+}\left|S_{R}\right|+\log A
$$

for the given $z$.

For convenience, we set

$$
\begin{aligned}
& g_{j-1}(z):=Q_{r_{j}^{0}}(f(z)), \quad j \in\{1, \ldots, n+1\}, \\
& g_{n+j}(z):=T_{j}(f)(z), \quad j \in\{1, \cdots, M-n\} .
\end{aligned}
$$

Noting that all $a_{i I}(z)$ are $c$-periodic functions, we have

$$
\begin{aligned}
& B_{0}(f)=\left|\begin{array}{cccccc}
Q_{r_{1}^{0}}(f) & \cdots & Q_{r_{n+1}^{0}}(f) & T_{1}(f) & \cdots & T_{M-n}(f) \\
Q_{r_{1}^{0}}(\bar{f}) & \cdots & Q_{r_{n+1}^{0}}(\bar{f}) & T_{1}(\bar{f}) & \cdots & T_{M-n}(\bar{f}) \\
\vdots & \vdots & \vdots & \vdots & \vdots & \vdots \\
Q_{r_{1}^{0}}\left(\bar{f}^{[M]}\right) & \cdots & Q_{r_{n+1}^{0}}\left(\bar{f}^{[M]}\right) & T_{1}\left(\bar{f}^{[M]}\right) & \cdots & T_{M-n}\left(\bar{f}^{[M]}\right)
\end{array}\right| \\
& =\left|\begin{array}{cccccc}
\frac{Q_{r_{1}^{0}}(f)}{Q_{r_{1}^{0}}(f)} & \cdots & \frac{Q_{r_{n+1}^{0}}(f)}{Q_{r_{n+1}^{0}}(f)} & \frac{T_{1}(f)}{T_{1}(f)} & \cdots & \frac{T_{M-n}(f)}{T_{M-n}(f)} \\
\vdots & \vdots & \vdots & \vdots & \vdots & \vdots \\
\frac{Q_{r_{1}^{0}}(f)}{[M]} & \cdots & \frac{Q_{r_{n+1}^{0}}(f)}{T_{1}(f)} & \frac{1 M}{T_{1}} & \ldots & \frac{T_{M-n}(f)}{[M]}
\end{array}\right|
\end{aligned}
$$

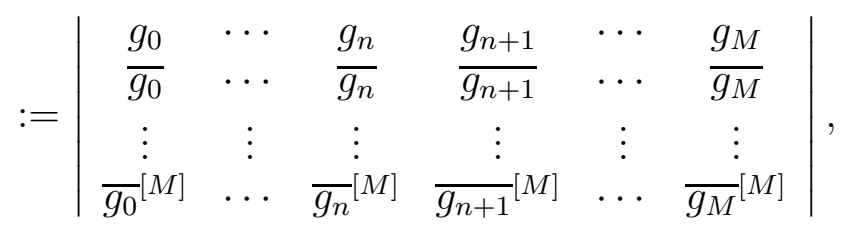


and thus

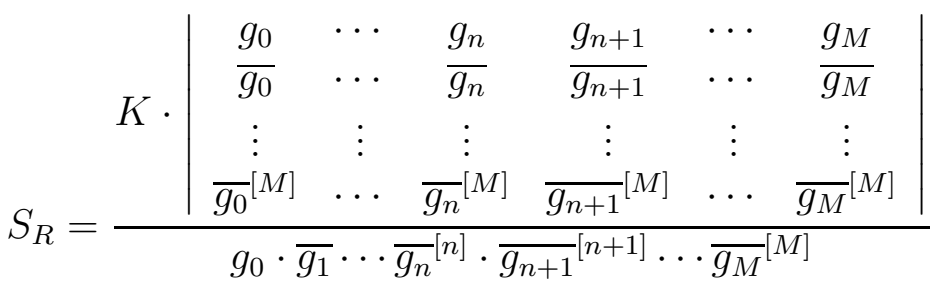

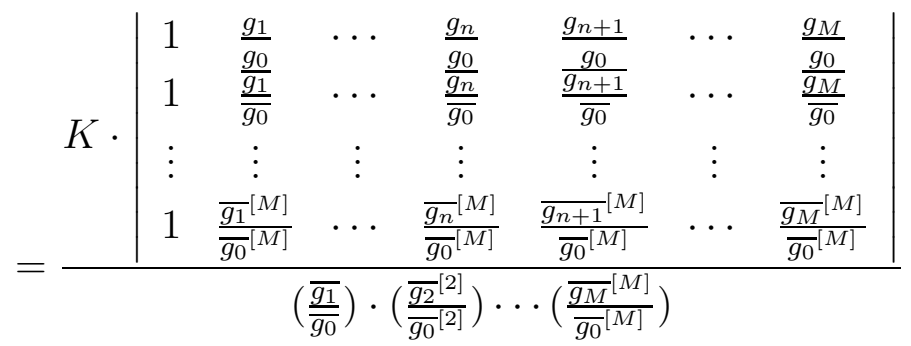

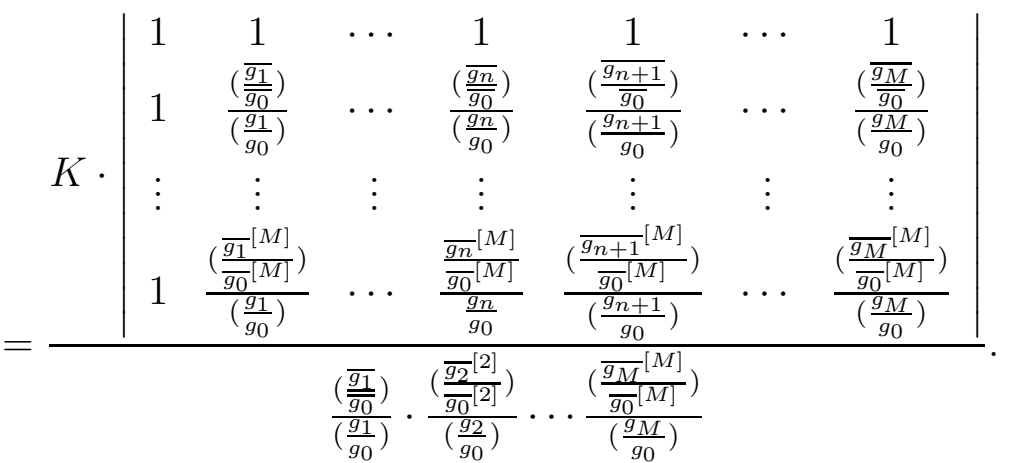

According to the characteristic function of holomorphic curves and noting that all coefficients $a_{i I}(z)$ of the moving hypersurfaces $\left\{Q_{i}(z)\right\}_{i=1}^{q}$ are slowly with respect to $f$, we have

$$
T\left(r, \frac{g_{i}}{g_{j}}\right) \leq d T_{f}(r)+o\left(T_{f}(r)\right), \quad i \neq j
$$

Then by the definition of hyperorder, we have $\varsigma_{2}\left(\frac{g_{i}}{g_{j}}\right) \leq \varsigma_{2}(f)<1$. Hence together with (3), and noting $K \in \mathcal{C}_{f}$, we can get from Lemma 3.1 that

$$
\frac{1}{2 \pi} \int_{0}^{2 \pi} \log ^{+}\left|S_{R}\left(r e^{\sqrt{-1} \theta}\right)\right| d \theta=o\left(\frac{T_{f}(r)}{r^{1-\varsigma_{2}(f)-\varepsilon}}\right)+o\left(T_{f}(r)\right)=o\left(T_{f}(r)\right)
$$

for all $r$ outside of a set of finite logarithmic measure $E$.

By the Jensen's Formula, we have

$$
\begin{aligned}
\int_{0}^{2 \pi} \log A \frac{d \theta}{2 \pi}= & \int_{0}^{2 \pi} \log \left\{\prod_{r_{i}^{0} \in R^{0}}\left|Q_{r_{i}^{0}}\left(\bar{f}^{[i-1]}(z)\right)\right| \prod_{i=1}^{M-n}\left|T_{i}\left(\bar{f}^{[n+i]}(z)\right)\right|\right\} \frac{d \theta}{2 \pi} \\
& -\int_{0}^{2 \pi} \log \left\{\prod_{r_{i}^{0} \in R^{0}}\left|Q_{r_{i}^{0}}(f(z))\right| \prod_{i=1}^{M-n}\left|T_{i}(f(z))\right|\right\} \frac{d \theta}{2 \pi}
\end{aligned}
$$




$$
\begin{aligned}
= & \sum_{r_{i}^{0} \in R^{0}} \int_{0}^{2 \pi} \log \left|Q_{r_{i}^{0}}\left(\bar{f}^{[i-1]}(z)\right)\right| \frac{d \theta}{2 \pi}+\sum_{i=1}^{M-n} \int_{0}^{2 \pi} \log \left|T_{i}\left(\bar{f}^{[n+i]}(z)\right)\right| \frac{d \theta}{2 \pi} \\
& -\sum_{r_{i}^{0} \in R^{0}} \int_{0}^{2 \pi} \log \left|Q_{r_{i}^{0}}(f(z))\right| \frac{d \theta}{2 \pi}-\sum_{i=1}^{M-n} \int_{0}^{2 \pi} \log \left|T_{i}(f(z))\right| \frac{d \theta}{2 \pi} \\
= & \sum_{r_{i}^{0} \in R^{0}} N\left(r, \frac{1}{Q_{r_{i}^{0}}\left(\bar{f}^{[i-1]}\right)}\right)+\sum_{i=1}^{M-n} N\left(r, \frac{1}{T_{i}\left(\bar{f}^{[n+i]}\right)}\right) \\
& -\sum_{r_{i}^{0} \in R^{0}} N\left(r, \frac{1}{Q_{r_{i}^{0}}(f)}\right)-\sum_{i=1}^{M-n} N\left(r, \frac{1}{T_{i}(f)}\right)+O(1) \\
= & \sum_{r_{i}^{0} \in R^{0}} N\left(r+(i-1)|c|, \frac{1}{Q_{r_{i}^{0}}(f)}\right)+\sum_{i=1}^{M-n} N\left(r+(n+i)|c|, \frac{1}{T_{i}(f)}\right) \\
& -\sum_{r_{i}^{0} \in R^{0}} N\left(r, \frac{1}{Q_{r_{i}^{0}}(f)}\right)-\sum_{i=1}^{M-n} N\left(r, \frac{1}{T_{i}(f)}\right)+O(1) .
\end{aligned}
$$

Furthermore, by the Jensen's Formula and the definition of characteristic function, we get that for any $j \in\{1,2, \ldots, q\}$,

$$
\begin{aligned}
N\left(r, \frac{1}{Q_{j}(f)}\right) & =\int_{0}^{2 \pi} \log \left|Q_{j}(f)\left(r e^{\sqrt{-1} \theta}\right)\right| \frac{d \theta}{2 \pi}+O(1) \\
& \leq \int_{0}^{2 \pi} \log \left\|f\left(r e^{\sqrt{-1} \theta}\right)\right\|^{d} \frac{d \theta}{2 \pi}+\int_{0}^{2 \pi} \log \left\|Q_{j}\left(r e^{\sqrt{-1} \theta}\right)\right\| \frac{d \theta}{2 \pi}+O(1) \\
& =d T_{f}(r)+o\left(T_{f}(r)\right),
\end{aligned}
$$

and thus

$$
\lambda_{2}\left(Q_{j}(f)\right)=\limsup _{r \rightarrow \infty} \frac{\log ^{+} \log ^{+} N\left(r, \frac{1}{Q_{j}(f)}\right)}{\log r} \leq \varsigma_{2}(f)<1 .
$$

Recall that from Lemma 3.5 we have

$$
\max _{j=1, \ldots, M-n}\left|T_{j}(f)(z)\right| \leq h_{3}(z)\|f(z)\|^{d},
$$

where $h_{3}(z) \in \mathcal{C}_{f}$. This gives from Jensen's Formula again that

$$
\begin{aligned}
N\left(r, \frac{1}{T_{j}(f)}\right) & =\int_{0}^{2 \pi} \log \left|T_{j}(f)\left(r e^{\sqrt{-1} \theta}\right)\right| \frac{d \theta}{2 \pi}+O(1) \\
& \leq \int_{0}^{2 \pi} \log \left\|f\left(r e^{\sqrt{-1} \theta}\right)\right\|^{d} \frac{d \theta}{2 \pi}+\int_{0}^{2 \pi} \log h_{3}\left(r e^{\sqrt{-1} \theta}\right) \frac{d \theta}{2 \pi}+O(1) \\
& =d T_{f}(r)+o\left(T_{f}(r)\right),
\end{aligned}
$$

and thus

$$
\lambda_{2}\left(T_{j}(f)\right)=\limsup _{r \rightarrow \infty} \frac{\log ^{+} \log ^{+} N\left(r, \frac{1}{T_{j}(f)}\right)}{\log r} \leq \varsigma_{2}(f)<1 .
$$


Hence by [9, Lemma 8.3], we get that for any $j \in\{1, \ldots, n+1\}$ and $i \in\{1, \ldots, M-n\}$,

$$
\begin{aligned}
N\left(r+(j-1)|c|, \frac{1}{Q_{r_{j}^{0}}(f)}\right) & \leq N\left(r, \frac{1}{Q_{r_{j}^{0}}(f)}\right)+o\left(\frac{N\left(r, \frac{1}{Q_{r_{j}^{0}}(f)}\right)}{r^{1-\lambda_{2}\left(Q_{r_{j}^{0}}(f)\right)-\varepsilon}}\right) \\
& \leq N\left(r, \frac{1}{Q_{r_{j}^{0}}(f)}\right)+o\left(T_{f}(r)\right)
\end{aligned}
$$

and

$$
\begin{aligned}
N\left(r+(i+n)|c|, \frac{1}{T_{i}(f)}\right) & \leq N\left(r, \frac{1}{T_{i}(f)}\right)+o\left(\frac{N\left(r, \frac{1}{T_{i}(f)}\right)}{r^{1-\lambda_{2}\left(T_{i}(f)\right)-\varepsilon}}\right) \\
& \leq N\left(r, \frac{1}{T_{i}(f)}\right)+o\left(T_{f}(r)\right) .
\end{aligned}
$$

Therefore, we obtain

$$
\int_{0}^{2 \pi} \log A\left(r e^{\sqrt{-1} \theta}\right) \frac{d \theta}{2 \pi}=o\left(T_{f}(r)\right)
$$

On the other hand,

$$
\begin{aligned}
& \int_{0}^{2 \pi} \log \left(\frac{\|\left. f\left(r e^{\sqrt{-1} \theta}\right)\right|^{d\left(\sum_{i=1}^{q} \omega_{i}\right)}|B(f)|}{\left.\left|Q_{1}\left(f\left(r e^{\sqrt{-1} \theta}\right)\right)\right|^{\omega_{1}} \cdots Q_{q}\left(f\left(r e^{\sqrt{-1} \theta}\right)\right)\right|^{\omega_{q}}}\right) \frac{d \theta}{2 \pi} \\
& =d\left(\sum_{j=1}^{q} \omega_{j}\right) \int_{0}^{2 \pi} \log || f\left(r e^{\sqrt{-1} \theta}\right)\left|\frac{d \theta}{2 \pi}+\int_{0}^{2 \pi} \log \right| B(f) \mid \frac{d \theta}{2 \pi} \\
& \quad-\sum_{j=1}^{q} \omega_{j} \int_{0}^{2 \pi} \log \left|Q_{j}(f)\left(r e^{\sqrt{-1} \theta}\right)\right| \frac{d \theta}{2 \pi}+O(1) \\
& =d\left(\sum_{j=1}^{q} \omega_{j}\right) \int_{0}^{2 \pi} \log || f\left(r e^{\sqrt{-1} \theta}\right)\left|\frac{d \theta}{2 \pi}+\int_{0}^{2 \pi} \log \right| \tilde{C}(f) \mid \frac{d \theta}{2 \pi} \\
& \quad+o\left(T_{f}(r)\right)-\sum_{j=1}^{q} \omega_{j} \int_{0}^{2 \pi} \log \left|Q_{j}(f)\left(r e^{\sqrt{-1} \theta}\right)\right| \frac{d \theta}{2 \pi} .
\end{aligned}
$$

Now combining this with (2), (4) and (5), we get from the definition of characteristic function and the Jensen's Formula that

$$
d\left(\sum_{j=1}^{q} \omega_{j}\right) T_{f}(r) \leq d(M+1) T_{f}(r)+\sum_{j=1}^{q} \omega_{j} N\left(r, \frac{1}{Q_{j}(f)}\right)-N\left(r, \frac{1}{\tilde{C}(f)}\right)+o\left(T_{f}(r)\right)
$$

for all $r \notin E$. By Lemma 3.3, we have $\omega_{j} \leq \tilde{\omega} \leq \frac{n}{N}$. Then the above inequality gives

$$
\begin{aligned}
& \left(q-\frac{(M+1)(2 N-n+1)}{n+1}\right) T_{f}(r) \\
& \leq \frac{1}{d} \sum_{j=1}^{q} N\left(r, \frac{1}{Q_{j}(f)}\right)-\frac{n}{d N} N\left(r, \frac{1}{\tilde{C}(f)}\right)+o\left(T_{f}(r)\right)
\end{aligned}
$$

for all $r \notin E$. 
We now prove the theorem in the general case where $\operatorname{deg} Q_{j}=d_{j}$. Note that hypersurfaces $Q^{\frac{d}{d_{j}}}(j=1, \cdots, q)$ have the common degree $d$. Then by the above discussion we can get

$$
\begin{aligned}
& \left(q-\frac{(M+1)(2 N-n+1)}{n+1}\right) T_{f}(r) \\
& \leq \frac{1}{d} \sum_{j=1}^{q} N\left(r, \frac{1}{Q_{j}^{\frac{d}{d_{j}}}(f)}\right)-\frac{n}{d N} N\left(r, \frac{1}{\tilde{C}(f)}+o\left(T_{f}(r)\right)\right. \\
& =\sum_{j=1}^{q} \frac{1}{d_{j}} N\left(r, \frac{1}{Q_{j}(f)}\right)-\frac{n}{d N} N\left(r, \frac{1}{\tilde{C}(f)}\right)+o\left(T_{f}(r)\right)
\end{aligned}
$$

for all $r \notin E$. Hence, we completely prove the theorem.

\section{Proof of Theorem 1.2}

Before proving Theorem 1.2, we introduce the following result due to Korhonen, Li and Tohge.

Lemma 5.1. [10] Let $f$ be a holomorphic curve of $\mathbf{C}$ into $\mathbf{P}^{n}(\mathbf{C})$, let $n \in \mathbf{N}$, $q>n$ and let

$$
a_{j}(z)=\left(a_{j 0}(z), \cdots, a_{j n}(z)\right), j \in\{1, \cdots, q\},
$$

where $a_{j k}(z)$ are c-periodic entire functions satisfying $T\left(r, a_{j k}(z)\right)=o\left(T_{f}(r)\right)$ for all $j, k \in\{1, \cdots, q\}$. If the (moving) hyperplanes

$$
H_{j}(z)=\left\{\left[x_{0}: \cdots: x_{n}\right]: \sum_{i=0}^{n} a_{j i} x_{i}=0\right\}, \quad j \in\{1, \cdots, q\},
$$

are located in general position. Then, we have

$$
\sum_{j=1}^{q} N\left(r, \frac{1}{H_{j}(f)(z)}\right)-N\left(r, \frac{1}{C(f)}\right) \leq \sum_{j=1}^{q} \tilde{N}_{f}^{[n, c]}\left(r, \frac{1}{H_{j}(f)(z)}\right)+o\left(T_{f}(r)\right) .
$$

Next, we give an extension of Lemma 5.1 to the case for moving hypersurfaces in $N$-subgeneral position.

Lemma 5.2. Let $f$ be a holomorphic curve of $\mathbf{C}$ into $\mathbf{P}^{n}(\mathbf{C})$. Let $\left\{Q_{j}(z)\right\}_{j=1}^{q}$ be c-periodic slowly moving hypersurfaces of $\mathbf{P}^{n}(\mathbf{C})$ with respect to $f$ in (weakly) $N$-subgeneral position with degree $d_{j}(1 \leq j \leq q)$. Let the least common multiple $d=\operatorname{lcm}\left(d_{1}, \cdots, d_{q}\right)$ and $M=\left(\begin{array}{l}n+d \\ d\end{array}\right)-1$. Then, we have

$$
\sum_{j=1}^{q} \frac{d}{d_{j}} N\left(r, \frac{1}{Q_{j}(f)}\right)-N\left(r, \frac{1}{\tilde{C}(f)}\right) \leq \sum_{j=1}^{q} \frac{d}{d_{j}} \tilde{N}_{f}^{[M, c]}\left(r, \frac{1}{Q_{j}(f)(z)}\right)+o\left(T_{f}(r)\right) .
$$

Proof. Firstly, we may assume that the homogeneous moving hypersurfaces $\left\{Q_{j}\right\}_{j=1}^{q}$ are homogeneous polynomials of degree $d$ in $N$-subgeneral position in $\mathbf{P}^{n}(\mathbf{C})$ in $\mathbf{C}\left[x_{0}, \cdots, x_{n}\right]$.

Since $\left\{Q_{j}(z)\right\}_{j=1}^{q}$ are in $N$-subgeneral position, we can find set $R \subset\{1,2, \cdots, q\}$ such that $N+1=\sharp R=\operatorname{rank}\left\{Q_{j}(z)\right\}_{j \in R}$. So we may assume $\operatorname{rank}\left\{Q_{j}(z)\right\}_{j=1}^{N+1}=N+1$. By Lemma 3.4, we can find $M-N$ moving hypersurfaces such that

$$
\operatorname{rank}\left\{\left\{Q_{j}(z)\right\}_{j=1}^{N+1} \cup\left\{T_{i}(z)\right\}_{i=N+2}^{M+1}\right\}=M+1 .
$$


Case 1: $N<M$. We may now assume that $z_{0}$ is an $M$-successive $c$-separated zero of $Q_{j}(f), 1 \leq j \leq N+1$. Now, there are integers $m_{j}(\geq 0)$ and holomorphic functions $h_{j k}(z)$ in a neighborhood $U$ of $z_{0}$ such that

$$
Q_{j}(f)(z+k c)=\left(z-z_{0}\right)^{m_{j}} h_{j k}(z),
$$

for $1 \leq j \leq N+1,0 \leq k \leq M$, and that

$$
T_{j}(f)(z+k c)=\left(z-z_{0}\right)^{m_{j}} h_{j k}(z),
$$

for $N+2 \leq j \leq M+1,0 \leq k \leq M$. Clearly, $m_{j}=0$ whenever either $Q_{j}(f)\left(z_{0}\right) \neq 0$ or $T_{j}(f)\left(z_{0}\right) \neq 0$. Write

$$
Q_{j}(f)(z)=\sum_{k=0}^{M} a_{j k}(z) f^{I_{k}}, T_{j}(f)(z)=\sum_{k=0}^{M} b_{j k}(z) f^{I_{k}},
$$

where $I_{k} \in \mathcal{J}_{d}, a_{j k}(z)$ and $b_{j k}$ are $c$-periodic entire functions and small with respect to $f$, then it follows that

$$
\begin{aligned}
A_{1}= & \left(\begin{array}{ccccccc}
Q_{1}(f) & Q_{2}(f) & \cdots & Q_{N+1}(f) & T_{N+2}(f) & \cdots & T_{M+1}(f) \\
Q_{1}(\bar{f}) & Q_{2}(\bar{f}) & \cdots & Q_{N+1}(\bar{f}) & T_{N+2}(\bar{f}) & \cdots & T_{M+1}(\bar{f}) \\
\vdots & \vdots & \vdots & \vdots & \vdots & \vdots & \vdots \\
Q_{1}\left(\bar{f}^{[M]}\right) & Q_{2}\left(\bar{f}^{[M]}\right) & \cdots & Q_{N+1}\left(\bar{f}^{[M]}\right) & T_{N+2}\left(\bar{f}^{[M]}\right) & \cdots & T_{M+1}\left(\bar{f}^{[M]}\right)
\end{array}\right), \\
A_{2}= & \left(\begin{array}{cccc}
a_{10}(z) & a_{11}(z) & \cdots & a_{1 M}(z) \\
\vdots & \vdots & \vdots & \vdots \\
a_{(N+1) 0}(z) & a_{(N+1) 1}(z) & \cdots & a_{(N+1) M}(z) \\
b_{(N+2) 0}(z) & b_{(N+2) 1}(z) & \cdots & \left.b_{(N+2) M}(z)\right) \\
\vdots & \vdots & \vdots & \vdots \\
b_{(M+1) 0}(z) & b_{(M+1) 1}(z) & \cdots & \left.b_{(M+1) M}(z)\right)
\end{array}\right),
\end{aligned}
$$

such that

$$
\operatorname{det} A_{1}=\tilde{C}(f) \cdot \operatorname{det} A_{2} .
$$

Since $\operatorname{rank}\left\{Q_{j}(z)\right\}_{j=1}^{N+1} \cup\left\{T_{i}(z)\right\}_{i=N+2}^{M+1}=M+1$, it is clear that $A_{2}$ is invertible and that $T\left(r,\left(\operatorname{det} A_{2}\right)^{-1}\right)=o\left(T_{f}(r)\right)$. The cases where $z_{0}$ is a zero of $\operatorname{det}\left(A_{2}^{-1}\right)$ can therefore be incorporated in the error term $o\left(T_{f}(r)\right)$. Hence, by (6) and (7) we have

$$
\tilde{C}(f)=\prod_{j=1}^{M}\left(z-z_{0}\right)^{m_{j}} h(z)
$$

where $h(z)$ is a holomorphic function defined on $U$. Thus $\tilde{C}(f)$ vanishes at $z_{0}$ with order at least $\sum_{j=1}^{q} m_{j}$. This, by going through all points $z_{0} \in \mathbf{C}$, together with definitions of $N\left(r, \frac{1}{Q_{j}(f)}\right), N\left(r, \frac{1}{\tilde{C}(f)}\right)$ and $\tilde{N}_{f}^{[M, c]}\left(r, \frac{1}{Q(f)(z)}\right)$ implies the assertion

$$
\sum_{j=1}^{q} N\left(r, \frac{1}{Q_{j}(f)(z)}\right)-N\left(r, \frac{1}{\tilde{C}(f)}\right) \leq \sum_{j=1}^{q} \tilde{N}_{f}^{[M, c]}\left(r, \frac{1}{Q_{j}(f)(z)}\right)+o\left(T_{f}(r)\right) .
$$

Case 2: $N \geq M$. Let $g_{k}:=f^{I_{k}}, 0 \leq k \leq M, I_{k} \in \mathcal{J}_{d}$, and $g:=\left[g_{0}: g_{1}: \cdots: g_{M}\right]$. Then $g$ is a holomorphic curve from $\mathbf{C} \rightarrow \mathbf{P}^{M}(\mathbf{C})$. According to the definition of the 
Nevanlinna-Cartan's characteristic function,

$$
\begin{aligned}
T_{g}(r) & =\int_{0}^{2 \pi} \log \max _{0 \leq j \leq M}\left\{\left|f^{I_{j}}\left(r e^{\sqrt{-1} \theta}\right)\right|\right\} \frac{d \theta}{2 \pi}+O(1) \\
& =\int_{0}^{2 \pi} \log \max _{0 \leq j \leq M}\left\{\left|g_{j}\left(r e^{\sqrt{-1} \theta}\right)\right|\right\} \frac{d \theta}{2 \pi}+O(1) \\
& \leq \int_{0}^{2 \pi} \log \max _{0 \leq j \leq n}\left\{\left|f_{j}\left(r e^{\sqrt{-1} \theta}\right)\right|^{d}\right\} \frac{d \theta}{2 \pi}+O(1) \\
& =d T_{f}(r)+O(1) .
\end{aligned}
$$

Set $H_{j}(g):=\sum_{k=0}^{M} a_{j k}(z) g_{k}$ for $j=1, \cdots, q$. Thus the moving hypersurfaces $\left\{Q_{j}(f)\right\}_{j=1}^{q}$ induce moving hyperplanes $\left\{H_{j}(f)\right\}_{j=1}^{q}$ which are of $M+1$ dimension. Now we can always find a set of hypersurfaces $\left\{Q_{j}\right\}_{j \in R}$ where $R \subset\{1,2, \cdots, q\}$ with $\sharp R=M+1=\operatorname{rank}\left\{Q_{j}\right\}_{j \in R}$, which induce moving hyperplanes $\left\{H_{j}\right\}_{j \in R}$. Then moving hyperplanes $\left\{H_{j}(z)\right\}_{j \in R}$ are in general position in $\mathbf{P}^{M}(\mathbf{C})$. By Lemma 5.1, we have

$$
\begin{aligned}
\sum_{j=1}^{q} N\left(r, \frac{1}{Q_{j}(f)(z)}\right)-N\left(r, \frac{1}{\tilde{C}(f)}\right) & =\sum_{j=1}^{q} N+\left(r, \frac{1}{H_{j}(g)(z)}\right)-N\left(r, \frac{1}{C(g)}\right) \\
& \leq \sum_{j=1}^{q} \tilde{N}_{g}^{[M, c]}\left(r, \frac{1}{H_{j}(g)(z)}\right)+o\left(T_{g}(r)\right) \\
& =\sum_{j=1}^{q} \tilde{N}_{f}^{[M, c]}\left(r, \frac{1}{Q_{j}(f)(z)}\right)+o\left(T_{f}(r)\right) .
\end{aligned}
$$

Secondly, we prove the lemma in the general case where $\operatorname{deg} Q_{j}=d_{j}$. Note that hypersurfaces $Q^{\frac{d}{d_{j}}}(j=1, \cdots, q)$ have the common degree $d$. From the above discussion, we have

$$
\sum_{j=1}^{q} N\left(r, \frac{1}{Q_{j}^{\frac{d}{d_{j}}}(f)}\right)-N\left(r, \frac{1}{\tilde{C}(f)}\right) \leq \sum_{j=1}^{q} \tilde{N}_{f}^{[M, c]}\left(r, \frac{1}{Q_{j}^{\frac{d}{d_{j}}}(f)(z)}\right)+o\left(T_{f}(r)\right)
$$

that is

$$
\sum_{j=1}^{q} \frac{d}{d_{j}} N\left(r, \frac{1}{Q_{j}(f)}\right)-N\left(r, \frac{1}{\tilde{C}(f)}\right) \leq \sum_{j=1}^{q} \frac{d}{d_{j}} \tilde{N}_{f}^{[M, c]}\left(r, \frac{1}{Q_{j}(f)(z)}\right)+o\left(T_{f}(r)\right) .
$$

Therefore, the lemma is proved.

The proof of Theorem 1.2. By Theorem 1.1 and Lemma 5.2 we have

$$
\begin{aligned}
& \left(q-\frac{(M+1)(2 N-n+1)}{n+1}\right) T_{f}(r) \\
& \leq \sum_{j=1}^{q} \frac{1}{d_{j}} N\left(r, \frac{1}{Q_{j}(f)}\right)-\frac{n}{d N} N\left(r, \frac{1}{\tilde{C}(f)}\right)+o\left(T_{f}(r)\right) \\
& \leq \sum_{j=1}^{q} \frac{1}{d_{j}} \tilde{N}_{f}^{[M, c]}\left(r, \frac{1}{Q_{j}(f)(z)}\right)+\frac{N-n}{d N} N\left(r, \frac{1}{\tilde{C}(f)}\right)+o\left(T_{f}(r)\right)
\end{aligned}
$$

for all $r \notin E$. 
Acknowledgement. The authors would like to thank the anonymous referee for giving many valuable suggestions and comments to improve this paper.

\section{References}

[1] An, D. P., S. D. QuAng, and D. D. ThAi: The second main theorem for meromorphic mappings into a complex projective space. - Acta Math. Vietnamica 38:1, 2013, 187-205.

[2] CAO, T. B.: Difference analogues of the second main theorem for meromorphic functions in several complex variables. - Math. Nachr. 287:5-6, 2014, 530-545.

[3] CaO, T. B., and R. J. Korhonen: A new version of the second main theorem for meromorphic mappings intersecting hyperplanes in several complex variables. - J. Math. Anal. Appl. 444:2, 2016, 1114-1132.

[4] Cartan, H.: Sur les zéros des combinaisons linéaires de p fonctions holomorphes donnés. Mathematica 7, 1933, 5-31.

[5] Chen, Z.X.: Complex differences and complex difference equations. Mathematics Monograph Deries 29, Science Press, Beijing, 2014.

[6] Chinng, Y.M., and S. J. Feng: On the Nevanlinna characteristic of $f(z+\eta)$ and difference equations in the complex plane. - Ramanujan J. 16, 2008, 105-129.

[7] Dethloff, G., and T. V. TAn: A second main theorem for moving hypersurface targets. Houston J. Math. 37, 2011, 79-111.

[8] Halburd, R. G., and R. J. Korhonen: Nevanlinna theory for the difference operator. - Ann. Acad. Sci. Fenn. Math. 31:2, 2006, 463-478.

[9] Halburd, R. G., R. J. Korhonen, and K. Tohge: Holomorphic curves with shift-invariant hyperplane preimages. - Trans. Amer. Math. Soc. 366:8, 2014, 4267-4298.

[10] Korhonen, R. J., N. Li, and K. Tohge: Difference analogue of Cartan's second main theorem for slowly moving periodic targets. - Ann. Acad. Sci. Fenn. Math. 41, 2016, 523-549.

[11] Nevanlinna, R.: Zur theorie der meromorphen funktionen. - Acta Math. 46:1-2, 1925, 1-99.

[12] NochkA, E. I.: On the theory of meromorphic functions. - Sov. Math. Dokl. 27, 1983, 377-381.

[13] Noguchi, J.: A note on entire presudo-holomorphic curves and the proof of Cartan-Nochka's theorem. - Kodai Math. J. 28, 2005, 336-346.

[14] Noguchi, J., and J. Winkelmann: Nevanlinna theory in several complex variables and diophantine approximation. - Springer, Japan, 2014.

[15] QuAng, S. D., and D. P. An: Second main theorem and unicity of meromorphic mappings for hypersurfaces in projective varieties. - arXiv:1412.1195v1, 2014.

[16] Ru, M.: Nevanlinna theory and its relation to diophatine approximation. - World Scientific Publishing Co., Singapore, 2001.

[17] Ru, M.: A defect relation for holomorphic curves intersecting hypersurfaces. - Amer. J. Math. 126, 2004, 215-226.

[18] Wong, P. M., H. F. LAw, and P. P. W. Wong: A second main theorem on $\mathbf{P}^{n}$ for difference operator. - Sci. China Ser. A 52:12, 2009, 2751-2758.

Received 9 November 2016 • Revised received 29 March 2017 • Accepted 31 March 2017 